\title{
EFICIÊNCIA DA ISCA MIREX-S (SULFURAMIDA 0,3\%) NO CONTROLE DE Atta cephalotes (HYMENOPTERA: FORMICIDAE) EM TRÊS DOSAGENS
}

\section{José Cola ZANUNCIO', Adalton Pinheiro da CRUZ ${ }^{2}$, Darcy Francisco dos SANTOS ${ }^{3}$, Marco Antonio de OLIVEIRA ${ }^{4}$}

RESUMO - Este trabalho foi realizado em reflorestamentos de eucalipto híbrido urograndis (Eucalyptus urophylla X Eucalyptus grandis) da Jari Celulose S.A, no município de Almerim, Pará, de julho de 1993 a janeiro de 1994. Objetivou-se testar a eficiência da isca granulada Mirex$\mathrm{S}$ (sulfluramida $0,3 \%$ ), nas dosagens de 6,8 e 10 gramas por metro quadrado de formigueiro, em comparação com 10 gramas de uma isca a base de dodecacloro $(0,45 \%)$ para o controle de Atta cephalotes (Hymenoptera: Formicidae). A isca Mirex-S apresentou índices de controle, respectivamente, de $72,70 \%, 83,33 \%$ e $91,67 \%$, em contrapartida com $87,50 \%$ da isca a base de dodecacloro. A isca Mirex-S, a 8 e 10 gramas por metro quadrado de formigueiro, pode substituir com eficiência as iscas à base de dodecacloro para o controle de A. cephalotes.

Palavras-Chave: Atta cephalotes, formigas cortadeiras, Mirex-S, Sulfluramida.

Efficiency of Mirex-S (Sulfluramid 0.3\%), in Three Dosages Against Atta cephalotes (Hymenoptera: Formicidae)

ABSTRACT - This study was developed in urograndis eucalypt hibrid (Eucalyptus urophylla $\mathrm{X}$ Eucalyptus grandis) plantation belonging to Jari Celulose S.A., in Monte Dourado, Pará State, Brazil, from July 1993 to January 1994. Mirex-S (sulfluramid $0.3 \%$ ) bait at the dosages of 6,8 and 10 grams was compared to 10 grams of a dodecachlor $(0.45 \%)$ bait per square meter of ant hill, against Atta cephalotes (Hymenoptera: Formicidae). At the dosages of 6,8 and 10 grams the Mirex-S bait showed $72.7 ; 83.3$ and $91.67 \%$ of nest control compared to $87.5 \%$ for the dodecachlor bait. Therefore, Mirex-S bait at 8 and 10 grams per square meter of ant hill can replace the dodecachlor baits for $A$. cephalotes control.

Key-words: Atta cephalotes, leaf cutting ants, Mirex-S, Sulfluramid.

\section{INTRODUÇÃO}

A formiga cortadeira Atta cephalotes (Hymenoptera:Formicidae), conhecida vulgarmente como saúva-da-mata, encontra-se distribuída nos Estados do Amazonas, Rondonia, Roraima, Pará, Amapá, Maranhão, Pernambuco (Recife e arredores) e Sul da Bahia, podendo ocorrer também no Acre e Norte do Mato Grosso (DELLA LUCIA et al., 1993). Esta espécie tem sido encontrada, também, nos plantios de
Eucalyptus da Jari Celulose, em Monte Dourado, Pará e vem sendo controlada por iscas granuladas à base de dodecacloro. Com a proibição deste clorado tomou-se necessária a busca de novos princípios ativos como a sulfluramida.

Os prejuízos causados pelas formigas cortadeiras aos setores agrícola e florestal intensificaram a busca de produtos e métodos de controle mais baratos, eficientes e menos agressivos ao meio ambiente. Embora a termonebulização possa ser

1 Universidade Federal de Viçosa. Departamento de Biologia Animal, 36571-000, Viçosa - MG.

2 Jari Celulose S.A. Almerin, 68240-000, Monte Dourado - Pará.

3 Universidade Federal de Viçosa. Departamento de Biologia Animal, 36571-000, Viçosa - MG. 
utilizada para o controle de formigas cortadeiras (COUTO et al., 1977; SANTOS et al., 1979) as iscas granuladas à base de dodecacloro (ZANUNCIO et al., 1980) ainda se constituem em um método indispensável, pela sua praticidade e menor custo. Com a recente proibição deste princípio ativo, surgiu a necessidade de testar-se novas substâncias para o controle de formigas.

Recentemente lançada, a isca Mirex-S, com o principio ativo sulfluramida $(0,3 \%)$ mostrou-se altamente eficiente para Atta laevigata (ZANUNCIO et al., 1992); Atta bisphaerica (ZANUNCIO et al., 1993a); Atta capiguara, Atta sexdens rubropilosa e A. laevigata (FORTI et al., 1993b); A. sexdens rubropilosa (LARANJEIRO \& ZANUNCIO, 1993; ZANUNCIO et al., 1993d); Acromyrmex crassispinus (ZANUNCIO et al., 1993c), Acromyrmex aspersus (CAETANO et al., 1993) e Acromyrmex subterraneus molestans (ZANUNCIO et al., 1993b).

A sulfluramida apresenta meia vida de 90 a 180 dias, em comparação com a meia vida de 12 anos do dodecacloro (PINHÃO et al., 1993). A morte das formigas ocorre devido a ação deste produto no processo de fosforilação oxidativa, interrompendo a produção de ATP (FORTI et al., 1993b).

Pela sua segurança, a sulfluramida é utilizada nos EUA para o controle de formigas em residências e, também, contra Solenopsis invicta (VANDER MEER et al., 1985; 1986) e Atta texana (CAMERON, 1990).

Pretende-se neste trabalho verificar a eficiência do controle de
Atta cephalotes (Hymenoptera: Formicidae) com a isca granulada Mirex-S (sulfluramida 0,3\%), em três dosagens, em comparação com uma isca à base de dodecacloro $(0,45 \%)$.

\section{Material e Métodos}

Este trabalho foi desenvolvido em plantios de eucalipto hibrido (Eucalyptus urophylla X Eucalyptus grandis) da Jari Celulose S.A. no Município de Almerim, Pará, de julho de 1993 a janeiro de 1994. Os produtos utilizados foram:

1. Nome comercial do produto: Mirex-S

Formulação: Isca granulada

Nome comum do princípio ativo: Sulfluramida

Concentração: 3 gramas de sulfluramida por quilograma de isca

Grupo químico: Sulfonamida fluoroalifática

2. Formulação: Isca granulada

Nome comum do princípio ativo: dodecacloro

Concentração: 4,5 gramas de dodecacloro por quilograma de isca

Grupo químico: Organoclorado

Nome químico: Dodecacloro octaidro 1,3,4 meteno $2 \mathrm{H}$ ciclobuta (cd) pentaleno (dodecacloro).

Os tratamentos utilizados foram:

Tratamento $\mathrm{T} 1-6$ gramas da isca Mirex-S por metro quadrado de terra solta sobre o formigueiro;

Tratamento $\mathrm{T} 2$ - 8 gramas da isca Mirex-S por metro quadrado de terra solta sobre o formigueiro;

Tratamento T3 - 10 gramas da isca Mirex-S por metro quadrado de terra solta sobre o formigueiro; 
Tratamento T4 - 10 gramas da isca a base de dodecacloro por metro quadrado de terra solta sobre o formigueiro;

Tratamento T5 - Testemunha.

Foram marcados 12 formigueiros para cada tratamento, mas durante a instalação deste experimento, alguns formigueiros que encontravam-se sem atividade foram descartados, ocasionando menor número de repetições em alguns tratamentos. Depois de terem suas áreas medidas (maior largura $\mathrm{X}$ maior comprimento de área de terra solta), e divididos em classes de tamanho os formigueiros receberam as quantidades de iscas determinadas pelos tratamentos, colocando-se toda a isca necessária nos olheiros de abastecimento, de uma só vez. A avaliação de carregamento e ou devolução foi feita 24 e 48 horas após a aplicação das iscas e as de atividade dos formigueiros observadas após $15,30,60,90,120$ e 150 dias da instalação do ensaio. Nesta última avaliação os formigueiros foram abertos com retroescavadeira e sondados com sonda JP, quando se determinou se estavam mortos ou não.

Os resultados foram analisados estatisticamente pelo teste de Duncan ao nível de $5 \%$ de probabilidade.

\section{RESULTADOS E DISCUSSÃO}

Durante a aplicação das iscas no campo observou-se uma intensa atividade de forrageamento de $A$. cephalotes e o transporte da isca em poucas horas. A maioria dos formigueiros não apresentou devolução das mesmas em qualquer das observações efetuadas. Isto demonstra que $A$. cephalotes pode ser, facilmente, controlada com iscas granuladas.

A eficiência de controle com a dosagem de 6 gramas da isca Mirex-S (sulfluramida $0,3 \%$ ) por metro quadrado de formigueiro, apresentou diferença significativa quando comparada com as dosagens de 8 e 10 gramas do mesmo produto e, também, para a isca à base de dodecacloro (Tab. 1). No entanto, a aplicação de 10 gramas da isca a base de dodecacloro e de 8 e 10 gramas da isca Mirex-S por metro quadrado de formigueiro não apresentou diferença estatística. A isca Mirex-S nas dosagens de 8 e 10 gramas pode substituir as iscas com dodecacloro no controle de A. cephalotes.

Tabela 1. Eficiência das iscas mirex-S (sulfluramida $0.3 \%$ ) e dodecacloro $(0,45 \%)$ no controle de Atta cephalotes (Hymenoptera: Formicidae), em Almerim, Monte Dourado, Pará.

\begin{tabular}{|c|c|c|c|c|c|c|c|c|}
\hline \multirow{4}{*}{$\begin{array}{l}\text { Trata- } \\
\text { mento }\end{array}$} & \multirow{4}{*}{ Isca } & \multirow{4}{*}{ Dosagem } & \multicolumn{2}{|c|}{ Formigueiros } & \multirow{3}{*}{ Carregamento } & & & \multirow{4}{*}{$\begin{array}{c}\text { Eficiència } \\
(\%)\end{array}$} \\
\hline & & & \multicolumn{2}{|l|}{ Area } & & \multirow{2}{*}{\multicolumn{2}{|c|}{ Formigueiros }} & \\
\hline & & & Mèdia & Amplitude & & & & \\
\hline & & & $m^{2}$ & $m^{2}$ & & Mortos & Vivos & \\
\hline$T 1$ & Mirex-S & $6 \mathrm{~g} / \mathrm{m}^{2}$ & 10.15 & $1.21-27.50$ & 68.75 & 8 & 3 & $72.70 \mathrm{~B}$ \\
\hline T2 & Mirex-S & $8 \mathrm{~g} / \mathrm{m}^{2}$ & 7.85 & $1.54-26.84$ & 88.33 & 10 & 2 & $83.33 \mathrm{~A}$ \\
\hline T3 & Mirex-S & $10 \mathrm{~g} / \mathrm{m}^{2}$ & 7.95 & $1.12-21.84$ & 85.56 & 10 & 1 & $90.09 \mathrm{~A}$ \\
\hline T4 & Dodecacloro & $10 \mathrm{~g} / \mathrm{m}^{2}$ & 9.85 & $2.75-28.00$ & 94.00 & 7 & 1 & $87.50 \mathrm{~A}$ \\
\hline T5 & Testemunha & $\ldots .$. & 7.04 & $1.50-25.38$ & ..... & 0 & 12 & $0.00 \mathrm{C}$ \\
\hline
\end{tabular}

Valores percentuais seguidos de uma mesma letra, não diferem entre si, pelo teste de Duncan, a $5 \%$ de probabilidade 
Exceto para o tratamento com 6 gramas da isca Mirex-S, que apresentou menor eficiência, os tratamentos com $8 \mathrm{e}$ 10 gramas desta isca e de 10 gramas da isca à base de dodecacloro, apresentaram respectivamente, $91,7 \%, 100 \%$ e $100 \%$ de formigueiros inativos aos 15 dias após a aplicação das mesmas (Fig. 1). Esta rápida inativação comprova os resultados obtidos em laboratório por FORTI et al. (1993b) para formigueiros com um ano de idade de $A$. sexdens rubropilosa onde, no terceiro e quarto dias após aplicação, as operárias pararam de cortar folhas, seguindo-se a desorganização da cultura do fungo, com grande crescimento micelial e a morte da rainha após 18 dias. Isto demonstra que a isca Mirex-S pode inativar, em definitivo, ou diminuir a atividade de forrageamento dos formigueiros, gradativamente, até a morte, de forma semelhante àquela das iscas à base de dodecacloro.

Os formigueiros que estavam inativados após 15 dias, continuaram da mesma forma até a avaliação final aos 150 dias, quando constatou-se que alguns encontravam-se, apenas, "amuados" o que pode estar relacionado com a distribuição da isca nas panelas de fungo. Isto demonstra a necessidade de avaliações minuciosas e a padronização de ensaios com iscas granuladas conforme sugerido por FORTI et al. (1993a), principalmente no que diz respeito ao período final de avaliação e na divisão dos formigueiros por classes de tamanho.

\section{CONCLUSÃO}

Dosagens de 8 e 10 gramas da isca Mirex-S (sulfluramida 0,3\%) por metro quadrado de terra solta sobre o

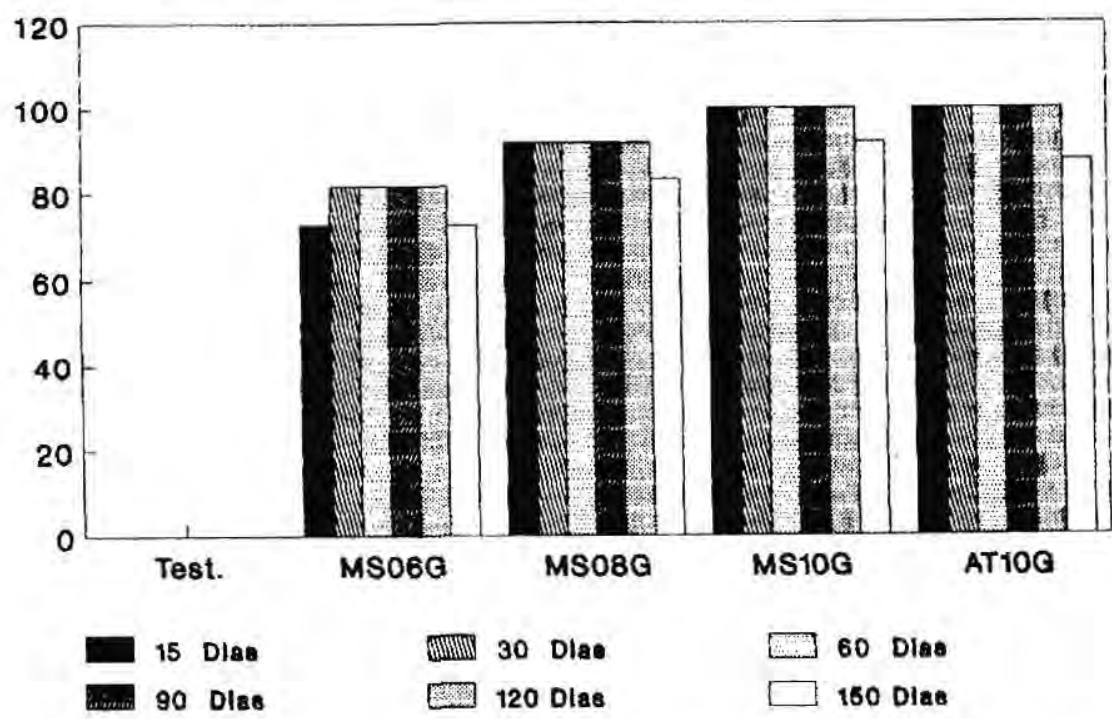

Figura 1. Percentagem de formigueiros de Atta cephalotes (Hymenoptera: Formicidae) paralisados aos 15, 30, 60, 90, 120 e 150 dias após a aplicação das iscas Mirex-S (MSO6G, MSO8G e MS10G, respectivamente, 6,8 , e 10 gramas da isca Mirex-S por metro quadrado de formigueiro) e à base de dodecacloro (ATIOG). Almerim, Monte Dourado, Pará. 
formigueiro, apresentaram aceitação e eficiência comparáveis àquelas da isca à base de dodecacloro $(0,45 \%)$. Por isto, recomenda-se a substituição das iscas à base de dodecacloro pela isca Mirex-S, no controle de $A$. cephalotes, nas dosagens de 8 e 10 gramas de isca por metro quadrado de formigueiro.

\section{Agradecimentos}

Ao Conselho Nacional de Desenvolvimento Científico eTecnológico $(\mathrm{CNPq})$ e à Fundação de Amparo à Pesquisa do Estado de Minas Gerais (FAPEMIG) pelas bolsas e auxílios concedidos. À Jari Celulose S.A. e à Sociedade de Investigações Florestais (SIF), através do Programa Cooperativo de Manejo Integrado de Pragas Florestais (PCMIP), pelo apoio para a realização desta pesquisa.

\section{Bibliografia Citada}

CAETANO, F.H.; PACHECO, P.; COSTACOELHO, L.C. 1993. Verificação da ação do Sulfluramid-GX-07IHB, isca granulada formicida sobre colônias de Acromyrmex aspersus (Hymenoptera: Formicidae). Anais do $14^{\circ}$ Congresso Brasileiro de Entomologia, Sociedade Entomológica do Brasil, Piracicaba, São Paulo, p.517.

CAMERON, R.S. 1990. Potential baits for control of the Texas leaf cutting ant, Atta texana (Hymenoptera: Formicidae). In: VANDER MEER, R.K.; JAFFÉ, K; CEDENO, A. (eds). Applied Myrmecology a world perspective, p. 628-37.

COUTO, L.; ZANUNCIO, J.C.; ALVES, J.E.M.; CAMPINHOS, E.; SORESINI, L.; VARGAS, J.A. 1977. Avaliação de eficiência de controle de Atta sexdens rubropilosa através do sistema de termonebulização, na Aracruz, ES. Revista Árvore, 1(1):9-16.

DELLA LUCIA, T.M.C.; FOWLER, H.G.;
MOREIRA, D.D.O. 1993. Espécies de formigas cortadeiras no Brasil. In: DELLA LUCIA, T.M.C. (ed.). As Formigas Cortadeiras. Viçosa, MG. p. 26-31

FORTI, L.C.; DELLA LUCIA, T.M.C.; YASSU, W.K.; BENTO, J.M.S.; PINHĀO, M.A.S. 1993a. Metodologias para experimentos com iscas granuladas para formigas cortadeiras. In: DELLA LUCIA, T.M.C. (ed). As Formigas Cortadeiras. Viçosa, MG. p.191-211.

FORTI, L.C.; PINHÃO, M.A.S.; YASSU, W.K.; PRETTO, D.R.; NAGAMOTO, N.S. 1993b. Pesquisas com Sulfluramida, no período de 1989 a 1993, para o controle de Atta spp. Anais do IV International Symposium on Pest Ants - XI Encontro de Mirmecologia. Belo Horizonte, MG, p.23.

LARANJEIRO, A.J.; ZANUNCIO, J.C. 1993. Avaliação da isca a base de sulfluramida no controle de Atta sexdens rubropilosa (Hymenoptera: Formicidae) pelo processo dosagem única de aplicaçōes de iscas formicidas. Anais do IV International Symposium on Pest Ants - XI Encontro de Mirmecologia. Belo Horizonte, MG., p.71.

PINHÃO, M.A.S.; FORTI, L.C.; YASSU, W.K.; NAGAMOTO, N.S. 1993. Mirex-S (sulfluramid): uma sulfona fluoroaliftica para o controle de Atta (Hymenoptera: Formicidae). Anais do $14^{\circ}$ Congresso Brasileiro de Entomologia. Sociedade Entomológica do Brasil, Piracicaba, São Paulo, p.511.

SANTOS, G.P.; GOMES, J.M.: ZANUNCIO, J.C.; BRANDI, R.M. 1979. Controle de saúvas pelo sistema de termonebulização na região de Timóteo, MG. Brasil Florestal, 9(38):18-20.

VANDER MEER, R.K.; LOFGREEN, C.S.; WILLIAMS, D.F. 1985. Fluoroaliphatic sulfones: A new class of delayed-action insecticides for control of Solenopsis invicta (Hymenoptera: Formicidae). J. Econ. Entomol., 78:1190-97.

VANDER MEER, R. K.; LOFGREEN, C. S.; WILLIAMS, D. F. 1986. Control of 
Solenopsis invicta with delayed-action fluornated toxicants. Pestic. Sci., 17:449-455.

ZANUNCIO, J.C.; VILELA, E.F; NOGUEIRA, S.B. 1980. Emprego de iscas granuladas e pós secos no controle de Atta Laevigata, no município de Curvelo, $\mathrm{MG}$. Revista Árvore, 4(2):221-6.

ZANUNCIO, J.C.; COUTO, L.; SANTOS, G.P.; ZANUNCIO, T.V. 1992. Eficiência da isca granulada Mirex-S, à base de Sulfluramida, no controle da formiga cortadeira Atta laevigata (Hymenoptera: Formicidae), Revista Ánore, 16(3): 357-361.

ZANUNCIO, J.C.; COUTO,L; FAGUNDES. M.; ZANUNCIO, T.V. 1993a, Eficiência da isca Mirex-S (sulfluramida $0,3 \%$ ) no controle da formiga cortadeira Atta bisphaerica Forel (Hymenoptera: Formicidae), Revista Árvore, 17(1):85-90.

ZANUNCIO, J.C.; LARANJEIRO, A.J.; SOUZA, O.F.F. 1993b. Avaliação da isca à base de sulfluramida no controle de Acromyrmex subterraneus molestans (Hymenoptera: Formicidae), na região de Aracruz, Espírito Santo. Anais do IV International Symposium on Pest Ants XI Encontro de Mirmecologia, Belo Horizonte, MG., p. 103.
ZANUNCIO, J.C.; RODRIGUES, F.A.; FAGUNDES, M.; BORESTAINER, S. 1993c. Eficiência da isca Mirex-S (sulfluramid $0,3 \%$ ) no controle de Acromyrmex crassispinus (Hymenoptera: Formicidae). Anais do $14^{\circ}$ Congresso Brasileiro de Entomologia. Sociedade Entomológica do Brasil, Piracicaba, SP. p.696.

ZANUNCIO, J.C.; SANTOS, G.P.; FIRME, J.D. 1993d. Avaliação da eficiência da isca granulada Mirex-S (sulfluramida $0,3 \%$ ) em Atta sexdens rubropilosa Forel, 1908 (Hymenoptera: Formicidae). Anais do IV International Symposium on Pest Ants - XI Encontro de Mirmecologia, Belo Horizonte, MG., p.102.

ZANUNCIO, J.C.; ZANUNCIO, T.V.; SANTOS,G.P. 1993e. A contribuição da pesquisa, em entomologia florestal, para a redução dos impactos ambientais dos reflorestamentos. Anais do Simpósio Brasileiro de Pesquisa Florestal, Belo Horizonte, MG., (1):136-142. 